\title{
Recalculation with SEACAB of the activation by spent fuel neutrons and residual dose originated in the racks replaced at Cofrentes NPP
}

\author{
Pedro Ortego ${ }^{1, a}$, Alain Rodriguez ${ }^{1}$, Candan Töre ${ }^{1}$, \\ José Luis de Diego Compadre ${ }^{2}$, Baltasar Rodriguez Quesada ${ }^{2}$, Raul Orive Moreno ${ }^{2}$ \\ ${ }^{1}$ SEA Ingeniería y Análisis de Blindajes S.L., Av. Atenas 75, Las Rozas 28232, Madrid, Spain \\ ${ }^{2}$ IBERDROLA Generación Nuclear S.A.U., Cofrentes NPP, Valencia, Spain
}

\begin{abstract}
In order to increase the storage capacity of the East Spent Fuel Pool at the Cofrentes NPP, located in Valencia province, Spain, the existing storage stainless steel racks were replaced by a new design of compact borated stainless steel racks allowing a $65 \%$ increase in fuel storing capacity. Calculation of the activation of the used racks was successfully performed with the use of MCNP4B code. Additionally the dose rate at contact with a row of racks in standing position and behind a wall of shielding material has been calculated using MCNP4B code as well. These results allowed a preliminary definition of the burnker required for the storage of racks. Recently the activity in the racks has been recalculated with SEACAB system which combines the mesh tally of MCNP codes with the activation code ACAB, applying the rigorous two-step method (R2S) developed at home, benchmarked with FNG irradiation experiments and usually applied in fusion calculations for ITER project.
\end{abstract}

\section{Introduction}

The reracking of a spent fuel pool is a frequent need in the effort to increase wet storage capacity of nuclear power plants. Cofrentes NPP, is a General Electric BWR-6 Mark III located in Valencia province, Spain, having two different wet storage ponds. In order to increase the storage capacity of the East Fuel Storage Pond (PACE in Spanish) the existing stainless steel racks were replaced by a new design of compact borated stainless steel racks allowing a $65 \%$ increase in fuel storing capacity.

More details about the rack characteristics, capacity improvement, extraction and installation procedure, etc, can be found in a previous paper [1]. At the removal of the old racks a decontamination process was performed eliminating completely the removable contamination but the intrinsic activity due to neutron irradiation needed to be estimated for designing the shielding of its interim storage at the plant.

The existing racks were irradiated by the residual neutron source originated in the $(\mathrm{a}, \mathrm{n})$ reactions and more important in the spontaneous fission of $\mathrm{Cm} 242$ and $\mathrm{Cm} 244$ isotopes.

The activation of the stainless steel rack channel had been calculated with the use of MCNP4B code using different libraries based on ENDF/B-IV and VI cross section evaluations. The neutron source was calculated with the use of the traditional SAS2H sequence inside the 4.4 version of SCALE [2].

Recently the activation has been recalculated with the use of SEACAB, a home developed system which couples the neutron flux map provided by the MCNP family of codes with the activation code ACAB. This system performs in an automated manner the activation and residual dose calculation according to the rigorous two-step method.

The final purpose of the activation calculation is the determination of the total Co60 photon source in the used racks to allow the design of a specific bunker inside the Almacén de Piezas de Baja Actividad o APBA (Low Activity Pieces Store), located at Cofrentes site.

Section 2 describes the estimation of a representative burnup and the activation calculation. Section 3 presents the dose estimates and Section 4 presents the conclusions.

\section{CALCULATION PROCESS}

The calculations of the spent fuel channel activation followed three different phases. First a representative and enveloping value of the fuel burnup was obtained by a detailed analysis of the management of the spent fuel pool positions during the previous 24 years of plant operation. Second the neutron source corresponding to such representative burnup was obtained assuming the typical irradiation cycle and the physical details of ATRIUM-10XP assembly design as enveloping of other fuel designs. Third the channel activation has been obtained with the use of MCNP4B [3] assuming a uniform neutron flux along the channel surface. Finally the activation has been recalculated with the use of SEACAB system and the results are compared with previous calculation results.

Additionally and in order to allow performing activation checks the photon dose in air at certain distances of the standing rack and of piled-up pieces of the rack structure have been calculated.

\footnotetext{
$\overline{\mathrm{a}}$ Corresponding author: p.ortego@seaingenieria.es
} 


\subsection{Estimation of fuel assembly burnup}

Cofrentes NPP keeps a complete record of the identity and characteristics of the fuel assembly located in each rack position. Nevertheless the calculation of the channel irradiation is a huge task due the large number of rack positions (1800), to the consideration of a detailed irradiation for each position and last but not least for the neutron cross talk between neighbour positions with different irradiation histories.

In general the rack positions have been occupied by the same assembly for long periods and the position has been modified occasionally at time periods associated to the refuelling outages, i.e. every 2 years as usual in the last few cycles. Therefore it is reasonable to assume that all occupied positions have reached the equilibrium in Co60 activity.

Concerning the photon source, only the peripheral positions of every rack are considered since the racks are planned to be stored in upright standing positions. The self-shielding provided by the $5 \mathrm{~mm}$ stainless steel rack channels is considered large enough to rapidly reduce the relative importance of the inner channels.

Due to the high variation of the neutron source intensity according to the assembly burnup, the simple average of assembly burnup is not a correct procedure for determining a representative burnup value. Therefore the results of SAS2 calculations performed for different burnup values have been fitted to a polynomial expression of order 3.4 .

The empty positions created a difficulty for estimating the average burnup since ignoring them meant to assume they had an assembly of the average burnup while giving a value of zero meant to neglect that they had been irradiated in previous time periods. Therefore the decay of the Co60 activity in the period when the rack position was empty was converted neutron intensity and further to a "reduced assembly burnup" by using the described expression, leading to the concept of "equivalent burnup".

The average values of equivalent burnup at the peripheral positions of the existing racks are presented in Table 1 where the left column corresponds to the assembly racks located at the North side of the PACE pond. It is seen that the range of burnups is not very wide and it can be stated that an enveloping value for the whole pond of $32 \mathrm{GWd} / \mathrm{tU}$ is reasonable. Using this enveloping value instead of the arithmetic average of about $24 \mathrm{GWd} / \mathrm{tU}$ means multiplying the source by 2 , given shape of the shape of the polynomial expression.

Table 1. Average value of equivalent burnup by rack.

\begin{tabular}{|c|c|c|c|c|c|}
\hline \multicolumn{6}{|c|}{ Burnup in North-South direction (GWd/tU) } \\
\hline N/A & N/A & 25.3 & 27.9 & 27.7 & 28.1 \\
\hline 20.6 & 22.7 & 22.6 & 22.2 & 22.4 & 22.2 \\
\hline 30.5 & 30.8 & 31.3 & 30.6 & 30.5 & 30.0 \\
\hline 25.8 & 25.9 & 25.4 & 20.1 & 26.7 & 25.8 \\
\hline
\end{tabular}

\subsection{Activation of the rack channel}

As indicated above, the neutron source at the assembly rods has been obtained with SAS2 sequence of SCALE4.4. As representative fuel assembly the $10 \times 10$ ATRIUM-10XP has been used for having a similar uranium mass to the GE14 and higher than the SVEA Optima2 fuel design. The irradiation in core during cycles 13 to $15 \mathrm{~B}$ has been modelled with 18 month cycles including 30 days of refuelling outage period. In these conditions the average neutron source per assembly is obtained as $1.34 \mathrm{E}+08$ neutron/s.

As the rack channel completely contours the fuel assembly, a single value of material activation has been obtained. This has been done with the FM card capability of MCNP4B and using different cross sections, including LLLDOS and ENDF601 based on ENDF/B-IV and VI respectively. The neutrons have been born uniformly inside each pellet volume and uniformly in the vertical direction. The model considers each individual rod and distinguishes pellet, gap and clad for each rod.

The only available information on the cobalt impurity content corresponded to a semi-quantitative analysis performed on a sample of the stainless steel material used in the West pond (Piscina Almacenamiento Combustible Oeste) being $0.25 \%$ or $2500 \mathrm{ppm}$. This value has been accepted for the PACE racks but as is obvious bears a large uncertainty.

The activation impact of an assembly occupying one position on the activation of the neighbour channels has been considered as well. The MCNP4B model is presented in Figure 1.
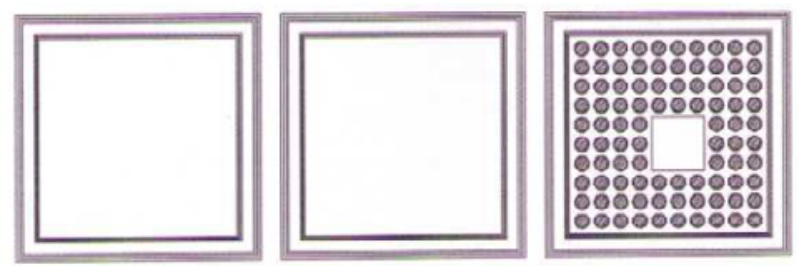

Figure 1 MCNP4B model of assembly and neighbour channels

\subsection{Activation calculations with MCNP4B}

The total specific activity per isotope in the enveloping rack channel is presented in Table 2 with the corresponding production reaction. It is seen that main activity corresponds to Co60 with $49.4 \mathrm{kBq} / \mathrm{cm} 3, \mathrm{Fe} 55$ with $48.9 \mathrm{kBq} / \mathrm{cm} 3$ and Ni63 with $22.1 \mathrm{kBq} / \mathrm{cm} 3$.

Concerning the mid-term storage of the racks only the Co60 is important. The total Co60 activity per channel is calculated as follows:

Channel volume $=2 \times 34 \times 430 \times 0.5=14525 \mathrm{~cm} 3$

Including the overlaping part of the bended plates making the channel up. The activity of each rack channel is then calculated as:

Activity $/$ channel $=14525 \times 430 \times 49400=7.18 \mathrm{E}+08 \mathrm{~Bq}$ 
and the total activity for a typical rack of $8 \times 10$ channels will be $5.7 \mathrm{E}+10$ and for the largest rack, with $10 \times 10$ channels, will be $7.2 \mathrm{E}+10 \mathrm{~Bq}$.

Table 2. Specific activity of different activation isotopes

\begin{tabular}{|c|c|c|}
\hline Active isotope & Main reaction & $\begin{array}{c}\text { Activity } \\
\text { (Bq/cm3) }\end{array}$ \\
\hline H3 & Li6(n,a)H3 & $4.08 \mathrm{E}+01$ \\
\hline C136 & Cl35(n,g)Cl36 & $1.94 \mathrm{E}+03$ \\
\hline Ca41 & Ca40(,n,g)Ca41 & $5.71 \mathrm{E}+00$ \\
\hline Mn54 & Fe54(n,p)Mn54 & $1.45 \mathrm{E}-03$ \\
\hline Ni59 & Ni58(n,g)Ni59 & $1.44 \mathrm{E}+05$ \\
\hline Co60 & Co59(n,g)Co60 & $4.94 \mathrm{E}+04$ \\
\hline Ni63 & Ni62(n,g)Ni63 & $2.21 \mathrm{E}+04$ \\
\hline Ag110m & Ag109(n,g)Ag110m & $4.59 \mathrm{E}+01$ \\
\hline Ag108m & Ag107(n,g)Ag108m & $1.20 \mathrm{E}+01$ \\
\hline Eu152 & Eu151(n,g)Eu152 & $1.28 \mathrm{E}+01$ \\
\hline Eu154 & Eu153(n,g)Eu154 & $7.10 \mathrm{E}-01$ \\
\hline Hf178m & Hf177(n,g)Hf178 & $1.40 \mathrm{E}+01$ \\
\hline Fe55 & Fe54(n,g)Fe55 & $4.89 \mathrm{E}+04$ \\
\hline C14 & N14(n,p)C14 & $3.83 \mathrm{E}+03$ \\
\hline Mo93 & Mo92(n,g)Mo93 & $5.31 \mathrm{E}+02$ \\
\hline
\end{tabular}

\subsection{Recalculations with SEACAB}

The SEACAB system has been benchmarked with the Frascati Neutron Generator irradiation and residual dose experiment [4].

The use of SEACAB requires the use of MCNPX or MCNP5 in order to obtain the detailed map of neutron flux in the whole energy spectrum. The so generated "mesh-tally" file is processed by SEACAB with an input file providing the fraction of material occupying each mesh volume. Additionally the irradiation and decay times are provided for their use in the activation-decayactivation cycle modelled by ACAB. Finally SEACAB gives the total activity in the channel and a distribution of activity point-by-point. This file is further used as SP card in the MCNP4B input to compute the dose rate at the position of interest as explained in next section.

\subsection{Dose calculations}

In order to advance the possible situations after racks extraction, it was necessary to provide the means to check the exact activation of each rack before its withdrawal from the pond. Therefore a set of photon dose calculations were performed by using the estimated activation to facilitate the comparison with the dose rate in air to be measured by Radiological Protection.

The dose in front of a row of standing racks has been calculated at short distance in conditions of direct view of a row of racks and behind different thicknesses of ordinary concrete and lead walls. The exact contribution of each row of channels has been calculated only for the first 3 rows, the dose contribution of the inner ones has been estimated with a row attenuation of $40 \%$ per row.

The dose rate obtained at contact is $4.58 \mathrm{mSv} / \mathrm{h}$. This value is larger than the maximum dose rate allowed inside the APBA, hence certain thickness of concrete or lead was necessary to reach the maximum dose rate corresponding to controlled area.

By using an ordinary concrete wall of $40 \mathrm{~cm}$ thickness the dose rate was calculated as $23 \mathrm{uSv} / \mathrm{h}$. By using a lead panel of $7 \mathrm{~cm}$ thickness the dose rate was calculated as $21 \mathrm{uSv} / \mathrm{h}$.

The measurements performed indicated that the calculated activity was overestimated with high probability due to the conservatism of both assembly burnup and cobalt content in the stainless steel.

\section{Summary and conclusions}

The calculation of the activation of the used racks has been successfully performed with the use of MCNP4B codes and the cross sections based on ENDF/B-IV and VI evaluated libraries.

Dose calculations have been performed with the use of MCNP4B and the activity of Co60 calculated. The dose at contact with a row of racks in standing positions and the dose behind different thickness of concrete and lead as shielding materials has been calculated. This has allowed the preliminary definition of the characteristics of the bunker required for the storage of racks.

Recently the activation of the channel and the corresponding dose rate in air has been recalculated with SEACAB system of codes. The results are compared successfully with the results obtained previously, taking into account the differences in assembly burnup and in other calculation hypotheses.

\section{References}

1. Nuclear España, Sociedad Nuclear Española Magazine, 298, July-August 2009

2. SCALE4.4

3. J.F. Briesmeister (Ed.), MCNP General Monte Carlo NParticle Transport Code, version $4 B$.

4. Fusion Engineering and Design 98-99 October 2015 pp 2214-2218. C. Töre, A. Rodriguez \& P. Ortego SEACAB qualification with Frascati Neutron Generator residual dose measurements 